EPJ manuscript No.

(will be inserted by the editor)

\title{
Accuracy of Auxiliary Field Approach for Baryons
}

\author{
I.M.Narodetskii ${ }^{1}$, C.Semay ${ }^{2}$, and A.I.Veselov ${ }^{1}$ a \\ 1 Institute of Theoretical and Experimental Physics, 117218 Moscow, Russia \\ ' 2 Groupe de Physique Nucléaire Théorique, Université de Mons-Hainaut, Place du Parc 20, BE-7000 Mons, Belgium.
}

Received: date / Revised version: date

\begin{abstract}
We provide a check of the accuracy of the auxiliary field formalism used to derive the Effective Hamiltonian for baryons in the Field Correlator Method. To this end we compare the solutions for the Effective Hamiltonian with those obtained from the solution of the spinless Salpeter equation. Comparing these results gives a first estimate of the systematic uncertainty due to the use of the auxiliary field formalism for baryons.
\end{abstract}

PACS. 12.38.-t Quantum chromodynamics - 12.40.Yx Hadron mass models and calculations

\section{Introduction}

The advent of new ideas concerning quark-quark forces in QCD has led to a revival of interest in baryon spectroscopy. Various versions of the constituent quark model [1] reproduce the octet and decuplet ground states but have very different and even contradictory predictions on the spectrum of excited states. It is therefore very important to develop model independent methods that are directly connected to the QCD Lagrangian and can help in alternatively understanding baryon spectroscopy.

One of such approaches is based on the Field Correlator Method (FCM) in QCD 2]. FCM provides a promising formulation of the nonperturbative QCD that gives additional support for the quark model assumptions. The application of this method for light mesons, heavy quarkonia, heavy-light mesons and light and heavy baryons can be found in Refs. [3]. The key ingredient of the FCM is the use of the auxiliary fields (AF) initially introduced in order to get rid of the square roots appearing in the relativistic Hamiltonian 1. Using the AF formalism allows one to write a simple local form of the Effective Hamiltonian $(\mathrm{EH})$ for the three quark system [6], which comprises both confinement and relativistic effects and contains only universal parameters: the string tension $\sigma$, the strong coupling constant $\alpha_{s}$, and the bare (current) quark masses $m_{i}$.

Send offprint requests to: I.M.Narodetskii

a This work was supported by RFBR grants 06-02-17120, 08-02-00657, and 08-02-00677. C. Semay thanks the F.R.S.FNRS for financial support.

1 Historically the AF formalism was first introduced in 4 to treat the kinematics of the relativistic spinless particles. For a brief review of the $\mathrm{AF}$ formalism relevant to the problem considered in this paper see Sec. II of [5].
The EH has the form

$$
H=\sum_{i=1}^{3}\left(\frac{m_{i}^{2}}{2 \mu_{i}}+\frac{\mu_{i}}{2}\right)+H_{0}+V .
$$

In Eq. (11), $H_{0}$ is the non-relativistic kinetic energy operator for masses $\mu_{i}, V$ is the sum of the string potential $V_{Y}\left(\mathbf{r}_{1}, \mathbf{r}_{2}, \mathbf{r}_{3}\right)$ and a Coulomb interaction term $V_{\text {Coulomb }}$ arising from the one-gluon exchange. The string potential is

$$
V_{Y}\left(\mathbf{r}_{1}, \mathbf{r}_{2}, \mathbf{r}_{3}\right)=\sigma r_{\min },
$$

where $r_{\min }$ is the minimal string length corresponding to the Y-shaped configuration. Finally the $\mu_{i}$ are the operator AF that have to be determined from the variational principle.

Note that the the sum of the mass term and $H_{0}$ in Eq. (11) can be conveniently written as

$$
\sum_{i=1}^{3}\left(\frac{m_{i}^{2}}{2 \mu_{i}}+\frac{\mu_{l}}{2}\right)+H_{0}=\sum_{i=1}^{3}\left(\frac{\mathbf{p}_{i}^{2}+m_{i}^{2}}{2 \mu_{i}}+\frac{\mu_{i}}{2}\right) .
$$

After taking the extremum of this expression in $\mu_{i}$ one ends with the standard relativistic kinetic energy operator $\sum_{i=1}^{3} \sqrt{\mathbf{p}_{i}^{2}+m_{i}^{2}}$

In this paper we use an approximate approach to consider the AF formalism first suggested in [7]. The AF are treated as c-number variational parameters. In this approach one replaces the operators $\mu_{i}(\tau)$ depending on time parameter $\tau$ by the c-numbers $\mu_{i}$ independent of $\tau$. The eigenvalue problem is solved for each set of $\mu_{i}$; then one has to minimize $\langle H\rangle$ with respect to $\mu_{i}$. Such an approach allows for a very transparent interpretation of AF: starting from bare quark masses $m_{i}$, we naturally arrive at the dynamical masses $\mu_{i}$ that appear due to the interaction 
and can be treated as the dynamical masses of constituent quarks.

An obvious disadvantage of the AF approach is that, as a variational method, it provides only an upper bound to the mass spectrum. So far the accuracy of this approximate solution for relativistic systems has been checked numerically only for mesons [5,8. The principle objective of this work is to test the AF method for baryons. We implement the AF method to calculate the baryon masses and then perform similar calculations using the relativistic Hamiltonian

$$
H=\sum_{i=1}^{3} \sqrt{\mathbf{p}_{i}^{2}+m_{i}^{2}}+V .
$$

Although being formally simpler the Hamiltonian (11) is equivalent to (4) up to the elimination of the AF (see e.g. Ref. 9]). We refer to an eigenvalue equation with Hamiltonian (4) as the spinless Salpeter equation (SSE). In QCD, it arises from the Bethe-Salpeter equation replacing the interaction by the instantaneous potential $V$ and considering a limited Fock space containing $q q q$ states only.

In this paper, we study the confinement plus Coulomb energies for the ground $S$-wave and orbitally excited $P$ wave states of nnn, nns and ssn baryons 2 and disregard the spin dependent forces, which are not relevant for our consideration.

The baryon masses in the AF approach are calculated using the hyperspherical method, while those in the SSE are calculated variationally. The numerical algorithm to solve the three-body problem variationally is based on an expansion of the wave function in terms of harmonic oscillator functions with different sizes [10]. The details of technical aspects can be found elsewhere [11. It was proved to give results of good accuracy if the expansion is pushed sufficiently far (let say up to 16-20 quanta). Moreover it can deal easily either with a non-relativistic or relativistic expression for the kinetic energy operator.

We find an accuracy of the AF method for hyperons to be about $6 \%$ at worst, which is quite reasonable to justify application of the AF formalism.

The paper is organized as follows. In Sec. 2, we briefly review the $\mathrm{EH}$ method. The application of this method for the baryons was described in detail elsewhere [12,13. Here we give only a brief summary important for our particular calculation. In Sec. 3, we discuss the hyperspherical approach, which is a very effective numerical tool to solve this Hamiltonian. In Sec.4, we provide a few numerical examples illustrating the accuracy of the hyperspherical solutions. In Sec. 5, predictions of the AF method are compared with those obtained from the solution of the spinless Salpeter equation (SSE). Section 6 contains our conclusions.

\footnotetext{
${ }^{2}$ Here and below the symbol $n$ stands for the light quarks $u$ or $d$.
}

\section{The baryon masses in the AF method and SSE}

The baryon mass in the FCM is given by

$$
\begin{gathered}
M_{B}^{A F}=M_{0}^{A F}+C^{A F} \\
M_{0}^{A F}=\sum_{i=1}^{3}\left(\frac{m_{i}^{2}}{2 \mu_{i}}+\frac{\mu_{i}}{2}\right)+E_{0}\left(\mu_{i}\right)
\end{gathered}
$$

where $E_{0}\left(\mu_{i}\right)$ is an eigenvalue of the Shrödinger operator $H_{0}+V$, the constant $\mathrm{AF} \mu_{i}$ are defined from the minimum condition

$$
\frac{\partial M_{0}^{A F}\left(m_{i}, \mu_{i}\right)}{\partial \mu_{i}}=0,
$$

and $C^{A F}$ is the quark self-energy correction which is created by the color magnetic moment of a quark propagating through the vacuum background field [14]. This correction, which can be added perturbatively, adds an overall negative constant to the hadron masses:

$$
C^{A F}=-\frac{2 \sigma}{\pi} \sum_{i} \frac{\eta\left(t_{i}\right)}{\mu_{i}}, \quad t_{i}=m_{i} / T_{g},
$$

where $1 / T_{g}$ is the gluonic correlation length. In what follows we use $T_{g}=1 \mathrm{GeV}$.

The function $\eta(t)$ is defined as

$$
\eta(t)=t \int_{0}^{\infty} z^{2} K_{1}(t z) e^{-z} d z,
$$

where $K_{1}$ is the McDonald function. A straightforward calculation yields [14]

$$
\begin{aligned}
\eta(t) & =\frac{1+2 t^{2}}{\left(1-t^{2}\right)^{2}}-\frac{3 t^{2}}{\left(1-t^{2}\right)^{5 / 2}} \ln \frac{1+\sqrt{1-t^{2}}}{t}, \quad t<1, \\
& \left.=\frac{1+2 t^{2}}{\left(1-t^{2}\right)^{2}}-\frac{3 t^{2}}{\left(t^{2}-1\right)^{5 / 2}} \arctan \left(\sqrt{t^{2}-1}\right), \quad t \ngtr 1 \mathbb{0}\right)
\end{aligned}
$$

Note that $\eta(0)=1$ and $\eta(t) \sim 2 / t^{2}$ as $t \rightarrow \infty$.

The baryon mass in the SSE approach is given by

$$
M_{B}^{S S E}=M_{0}^{S S E}+C^{S S E}
$$

where $M_{0}^{S S E}$ is an eigenvalue of the relativistic Hamiltonian (41) and the $C^{S S E}$ are given by (8) with the obvious substitution $\mu_{i} \rightarrow \omega_{i}$, where

$$
\omega_{i}=\left\langle\sqrt{\mathbf{p}_{i}^{2}+m_{i}^{2}}\right\rangle
$$

are the average kinetic energies of the current quarks.

We will not perform a systematic study in order to determine the best set of parameters to fit the baryon spectra. Instead, in what follows we employ some typical values of the string tension $\sigma$ and the strong coupling constant $\alpha_{s}$, which have been used for the description of the ground state baryons [12] $\sigma=0.15 \mathrm{GeV}^{2}$ and $\alpha_{s}=0.39$. In our calculations we use the values of the current light 
quark masses, $m_{u}=m_{d}=9 \mathrm{MeV}$, and $m_{s}=175 \mathrm{MeV}$. As in Ref. 12 we neglect the spin dependent potentials responsible for the fine and hyperfine splittings of baryon states.

Our aim is to compare the baryon masses given by Eqs. (5) and (11). To this end we first solve the non-relativistic Schrödinger equation with the confining and Coulomb interactions to determine the constituent quark masses $\mu_{i}$ and the baryon masses $M_{B}^{A F}$. Efficient methods to deal with the Y-shape interaction rely either on Monte-Carlo algorithms [15, 16] or the hyperspherical method [17. We use the latter approach.

\section{Outline of the hyperspherical formalism.}

In this section, we briefly review the hyperspherical method, which we use to calculate the masses of the ground and excited hyperon states.

The baryon wave function depends on the three-body Jacobi coordinates

$$
\begin{aligned}
\boldsymbol{\rho}_{i j} & =\sqrt{\frac{\mu_{i j}}{\mu_{0}}}\left(\boldsymbol{r}_{i}-\boldsymbol{r}_{j}\right), \\
\boldsymbol{\lambda}_{i j} & =\sqrt{\frac{\mu_{i j, k}}{\mu_{0}}}\left(\frac{\mu_{i} \boldsymbol{r}_{i}+\mu_{j} \boldsymbol{r}_{j}}{\mu_{i}+\mu_{j}}-\boldsymbol{r}_{k}\right),
\end{aligned}
$$

$\left(i, j, k\right.$ cyclic), where $\mu_{i j}$ and $\mu_{i j, k}$ are the appropriate reduced masses:

$$
\mu_{i j}=\frac{\mu_{i} \mu_{j}}{\mu_{i}+\mu_{j}}, \quad \mu_{i j, k}=\frac{\left(\mu_{i}+\mu_{j}\right) \mu_{k}}{\mu_{i}+\mu_{j}+\mu_{k}},
$$

and $\mu_{0}$ is an arbitrary parameter with the dimension of mass, which drops out in the final expressions. There are three equivalent ways of introducing the Jacobi coordinates, which are related to each other by linear transformations with the Jacobian equal to unity. In what follows we omit the indices $i$ and $j$.

In terms of the Jacobi coordinates the kinetic energy operator $H_{0}$ in (11) is written as

$$
\begin{aligned}
& H_{0}=-\frac{1}{2 \mu_{0}}\left(\frac{\partial^{2}}{\partial \boldsymbol{\rho}^{2}}+\frac{\partial^{2}}{\partial \boldsymbol{\lambda}^{2}}\right)= \\
& =-\frac{1}{2 \mu_{0}}\left(\frac{\partial^{2}}{\partial R^{2}}+\frac{5}{R} \frac{\partial}{\partial R}+\frac{\boldsymbol{L}^{2}(\Omega)}{R^{2}}\right),
\end{aligned}
$$

where $R$ is the six-dimensional hyperradius that is invariant under quark permutations,

$$
\begin{aligned}
& R^{2}=\rho^{2}+\lambda^{2}, \\
& \rho=R \sin \theta, \quad \lambda=R \cos \theta, \quad 0 \leq \theta \leq \pi / 2,
\end{aligned}
$$

$\Omega$ denotes five residuary angular coordinates, and $\boldsymbol{L}^{2}(\Omega)$ is an angular operator

$$
\mathbf{L}^{2}=\frac{\partial^{2}}{\partial \theta^{2}}+4 \cot \theta \frac{\partial}{\partial \theta}-\frac{\mathbf{l}_{\rho}^{2}}{\sin ^{2} \theta}-\frac{\mathbf{l}_{\lambda}^{2}}{\cos ^{2} \theta},
$$

whose eigenfunctions (the hyperspherical harmonics) satisfy

$$
\mathbf{L}^{2}(\Omega) Y_{[K]}\left(\theta, \mathbf{n}_{\rho}, \mathbf{n}_{\lambda}\right)=-K(K+4) Y_{[K]}\left(\theta, \mathbf{n}_{\rho}, \mathbf{n}_{\lambda}\right),
$$

with $K$ being the grand orbital momentum.

The wave function $\psi(\boldsymbol{\rho}, \boldsymbol{\lambda})$ is written in a symbolical shorthand as

$$
\psi(\boldsymbol{\rho}, \boldsymbol{\lambda})=\sum_{[K]} \psi_{[K]}(R) Y_{[K]}(\Omega),
$$

where the set $[K]$ is defined by the orbital momentum of the state and the symmetry properties.

We truncate this set using the approximation $K=$ $K_{\min }$. We comment on the accuracy of this approximation latter on. Our task is then extremely simple in principle: we have to choose a zero-order wave function corresponding to the minimal $K$ for a given $L\left(K_{\min }=0\right.$ for $L=0$ and $K_{\min }=1$ for $L=1$ ). The corresponding hyperspherical harmonics are

$$
\begin{aligned}
& Y_{0}=\sqrt{\frac{1}{\pi^{3}}}, \quad K=0, \\
& \boldsymbol{Y}_{\rho}=\sqrt{\frac{6}{\pi^{3}}} \frac{\boldsymbol{\rho}}{R}, \quad \boldsymbol{Y}_{\lambda}=\sqrt{\frac{6}{\pi^{3}}} \frac{\lambda}{R}, \quad K=1 .
\end{aligned}
$$

For nns baryons we use the basis in which the strange quark is singled out as quark 3 but in which the nonstrange quarks are still antisymmetrized. In the same way, for the ssn baryon we use the basis in which the non strange quark is singled out as quark 3 . The nns basis states diagonalize the confinement problem with eigenfunctions that correspond to separate excitations of the non-strange and strange quarks ( $\rho$ - and $\lambda$ excitations, respectively). In particular, excitation of the $\boldsymbol{\lambda}$ variable unlike excitation in $\boldsymbol{\rho}$ involves the excitation of the "odd" quark ( $s$ for $n n s$ or $n$ for $s s n$ ). The nonsymmetrized $u d s$ and $s s q$ bases usually provide a much simplified picture of the states. The physical P-wave states are neither pure $\mathrm{SU}(3)$ states nor pure $\rho$ or $\lambda$ excitations but linear combinations of all states with a given $J$. Most physical states are, however, closer to pure $\rho$ or $\lambda$ states than to pure $\mathrm{SU}(3)$ states [18. Note that for the $n n n$ baryons, the $\boldsymbol{\rho}$ and $\boldsymbol{\lambda}$ excitation energies are degenerate.

Introducing the reduced function $u_{\gamma}(R)$

$$
\Psi_{\gamma}(R, \Omega)=\frac{u_{\gamma}(R)}{R^{5 / 2}} \cdot Y_{\nu}(\Omega),
$$

where $\gamma=0$ for $L=0, \gamma=\rho, \lambda$ for $L=13$, the new variable

$$
\begin{aligned}
& x=\sqrt{\mu_{0}} R= \\
& \left(\sum_{i} \frac{\mu_{1} \mu_{2}}{M} r_{12}^{2}+\frac{\mu_{2} \mu_{3}}{M} r_{23}^{2}+\frac{\mu_{3} \mu_{1}}{M} r_{31}^{2}\right)^{1 / 2},
\end{aligned}
$$

\footnotetext{
3 In what follows, for ease of notation we will drop the magnetic quantum numbers of the vector spherical harmonics.
} 
and averaging the interaction $V=V_{Y}+V_{C}$ over the sixdimensional sphere $\Omega$ with the weight $\left|Y_{\gamma}\right|^{2}$, one obtains the one-dimensional Schrödinger equation for $u_{\gamma}(x)$

$$
\begin{aligned}
& \frac{d^{2} u_{\gamma}(x)}{d x^{2}}+ \\
& 2\left(E_{0}-\frac{\left(K+\frac{3}{2}\right)\left(K+\frac{5}{2}\right)}{2 x^{2}}-V_{\gamma}(x)\right) u_{\gamma}(x)=0,2
\end{aligned}
$$

where $V_{\gamma}(x)=V_{\mathrm{Y}}^{\gamma}(x)+V_{\text {Coulomb }}^{\gamma}(x)$,

$$
\begin{aligned}
& V_{\mathrm{Y}}^{\gamma}(x)=\int\left|Y_{\gamma}(\theta, \chi)\right|^{2} V_{\mathrm{Y}}\left(\mathbf{r}_{1}, \mathbf{r}_{2}, \mathbf{r}_{3}\right) d \Omega= \\
& \sigma b_{\nu} \frac{x}{\sqrt{\mu_{0}}},
\end{aligned}
$$

and

$$
\begin{aligned}
& V_{\text {Coulomb }}^{\gamma}(x)=-\frac{2}{3} \alpha_{s} \int\left|Y_{\gamma}(\theta, \chi)\right|^{2} \sum_{i<j} \frac{1}{r_{i j}} d \Omega= \\
& -\frac{2}{3} \alpha_{s} \frac{a_{\gamma}}{x} \sqrt{\mu_{0}} .
\end{aligned}
$$

In what follows we denote

$$
\mu_{1}=\mu_{2}=\mu, \quad \mu_{3}=\kappa \mu .
$$

Then the straightforward analytical calculation of the integrals in (25) yields

$$
\begin{gathered}
a_{0} \sqrt{\mu_{0}}=\frac{16}{3 \pi}\left(\sqrt{2}+2 \sqrt{\frac{\kappa}{1+\kappa}}\right) \sqrt{\mu}, \\
a_{\rho} \sqrt{\mu_{0}}=\frac{32}{15 \pi}\left(\sqrt{2}+\sqrt{\frac{\kappa}{1+\kappa}} \frac{5 \kappa+6}{1+\kappa}\right) \sqrt{\mu}, \\
a_{\lambda} \sqrt{\mu_{0}}=\frac{32}{15 \pi}\left(\frac{3}{\sqrt{2}}+\sqrt{\frac{\kappa}{1+\kappa}} \frac{4+5 \kappa}{1+\kappa}\right) \sqrt{\mu} .
\end{gathered}
$$

For $\kappa=1$ (the nnn system) $a_{\rho}=a_{\lambda}$. The corresponding expressions for $b_{\gamma}$ are more complicated (see, e.g., the appendix of Ref. [13]).

\section{Accuracy of the hyperspherical approximation}

A few words concerning the accuracy of the approximation $K=K_{\min }$ are in order. An illustration of the accuracy of the hyperspherical approximation $K=K_{\min }$ is given by the results presented in Table 1 . This Table compares the eigenvalues $E_{0}$ in Eq. (6) for the $n n n, n n s$ and $s s n$ systems obtained using the variational method and those calculated from Eq. (23) with $K=K_{\min }$ 4. In all cases the dynamical masses $\mu_{i}$ are the same as were found from the minimum condition (7) for the Y-shaped string potential [13. For technical reasons the variational calculations

\footnotetext{
${ }^{4}$ Recall that, as was stated in Sec. 3, the $\boldsymbol{\rho}$ and $\boldsymbol{\lambda}$ excitation energies for the $n n n$ baryon are degenerate.
}

have been performed not for the genuine string junction potential but for its approximation by a sum of the oneand two-body confining potentials [19]

$$
V_{\mathrm{M}}=\frac{1}{2}\left(V_{\Delta}+V_{\mathrm{CM}}\right)
$$

where $V_{\Delta}$ with the sum of the two-body confining potentials is

$$
V_{\Delta}=\sigma \frac{1}{2} \sum_{i<j} r_{i j}=\sigma \frac{1}{2} \sqrt{\mu_{0}} \sum_{i<j} \frac{\left|\boldsymbol{\rho}_{i j}\right|}{\sqrt{\mu_{i j}}},
$$

and $V_{\mathrm{C}}$ is the sum of one-body center-of-mass string potentials:

$$
\begin{aligned}
V_{\mathrm{CM}} & =\sigma \sum_{i}\left|\boldsymbol{r}_{i}-\boldsymbol{R}_{c m}\right| \\
& =\sigma \sqrt{\mu_{0}} \sum_{(i, j, k)} \frac{1}{\mu_{k}} \sqrt{\mu_{i j, k}}\left|\boldsymbol{\lambda}_{i j}\right|,
\end{aligned}
$$

( $i, j, k$ cyclic), where $\boldsymbol{R}_{c m}$ is the center-of-mass coordinate. Table 1 also compares eigenvalues $E_{0}^{Y}$ for the genuine string potential $V_{Y}$ with those for the confining potentials $V_{\Delta}, V_{\mathrm{C} M}$ and $V_{\mathrm{M}}$ with the same string tension. The confining potential $V_{\mathrm{C} M}$ overestimates the eigenvalues of the genuine string junction $E_{0}^{Y}$ while the potential $V_{\Delta}$ underestimates the $E_{0}^{Y}$, i.e. $E_{0}^{\Delta}<E_{0}^{Y}<E_{0}^{\mathrm{CM}}$ (compare columns 6,7 and 8 of Table 1). The values of the two columns 7 and 8 are in reasonable agreement with the reference results of column 6. In line with expectations [19, the eigenvalues for the genuine string junction change little if we use $V_{\mathrm{M}}$ instead of $V_{\mathrm{Y}}$. Simulation of the genuine string junction potential by a sum of the two-body confining potentials (30) (column 9) is a good approximation in all cases: using $V_{\mathrm{M}}$ results in a $\sim 20 \mathrm{MeV}$ or $1-2 \%$ downwards shift of $E_{0}$ for all states (compare columns 6 and 9). Let us note that Hamiltonian 1 with potential $V_{M}$ gives eigenvalues wich are, to some $\mathrm{MeV}$, the arithmetic mean of the eigenvalues with potential $V_{\Delta}$ and $V_{C M}$. So the contributions of $V_{\Delta}$ and $V_{C M}$ to $V_{M}$ are nearly evenly distributed.

The last column 10 contains the eigenvalues $E_{0 \text { var }}^{M}$ calculated using the variational method briefly described in Sect. 1. Comparing the column 9 and 10 of Table 1 we conclude that the hyperspherical and variational results are close enough to validate the approximation $K=K_{\min }$.

\section{Comparison of the AF and SSE results}

Table 2 compares the baryon masses computed using the $\mathrm{AF}$ and SSE formalisms. In this Table we list the masses of the nnn, nns and ssn states with $L=0,1$. The entries labeled $A F$ have been calculated from Eq. (23) with $K=K_{\text {min }}$, while the entries labeled $S S E$ have been calculated using the variational method for the relativistic Hamiltonian (4). In both cases, we approximate the Y shaped string potential by the expression (30). As was mentioned in the Introduction the comparison of the AF 
results with those evaluated from the solution of SSE has been performed only for the $\bar{q} q$ mesons with the conclusion that the variational AF method gives a systematic overestimation of order 5-7\% 5, 8ur calculations show the similar results: the relative deviation

$$
\varepsilon=\frac{M_{B}^{A F}-M_{B}^{S S E}}{M_{B}^{S S E}}
$$

is positive and for most considered states does not exceed $6 \% 5$. The accuracy of the AF approach does not seem to be very sensitive to the bare light-quark masses. The quantum numbers of states have a stronger influence on the accuracy. In particular, $\varepsilon$ for the $L=1$ states are uniformly smaller than those for the $L=0$ states. Curiously, the self-energy corrections $C^{M F C}$ and $M^{S S E}$ agree even with better accuracy (typically within $5 \%$ or even better) in spite of the fact that the difference $\mu_{i}$ and $\omega_{i}$ in some cases (e.g. for the $\lambda$ excitation in the ssn) comprises $30 \%$. As for the excitation energies, $\Delta=M_{B}(L=1)-M_{B}(L=0)$ evaluated using the AF and SSE methods, they practically coincide for the ssn baryons and differ no more than $\sim 30 \mathrm{MeV}$ for the nns baryons. Taking into consideration that we neglect the spin interactions the baryon energies calculated using SSE agree reasonably with the data 20]. For instance, for $L=0$ we get $\frac{1}{2}(N+\Delta)_{\text {theory }}=1062 \mathrm{MeV}$ versus $\frac{1}{2}(N+\Delta)_{\exp }=1085 \mathrm{MeV}$ and $\frac{1}{4}\left(\Lambda+\Sigma+2 \Sigma^{*}\right)_{\text {theory }}=$ $1220 \mathrm{MeV}$ versus $\frac{1}{4}\left(\Lambda+\Sigma+2 \Sigma^{*}\right)_{\exp }=1267 \mathrm{MeV} . \mathrm{A}$ similar correspondence exists for the other states considered in this work.

\section{Conclusions}

In this paper we have tested the quality of our previous study of the masses of the S- and P- baryon states obtained in the FCM with the use of the AF formalism. To this end we have compared the AF results with those obtained from the solution of the SSE with the same interaction. The main purpose was to check whether the results obtained within these two methods are similar. We have found that they agree within $\sim 100 \mathrm{MeV}$ for the absolute values of masses and with much better accuracy for the excitation energies. Thereby our study supports the AF basic assumptions by the compatibility of its mass predictions with the masses derived from the SSE. Moreover, this comparative study gives better insight into the quark model results, where the constituent masses encode the QCD dynamics.

\section{References}

1. S. Capstick and N. Isgur, Phys. Rev. D 34, (1986) 2809: L. Ya. Glozman and D. O. Riska, Phys. Rep. 268, (1996) 263.

\footnotetext{
${ }^{5}$ An obvious exception is the $n n n$ state with $L=0$ for which $\varepsilon$ reaches $14 \%$.
}

2. H. G. Dosch, Phys. Lett. 190, (1987) 177; H. G. Dosch, Yu. A. Simonov, Phys. Lett. 202, (1988) 339; Yu. A. Simonov, Nucl. Phys. B307, (1988) 512.

3. A. M. Badalian, B. L. G. Bakker, Yu. A. Simonov, e-print arXiv: hep-ph/0702157; I. M. Narodetskii, M .A .Trusov and A. I. Veselov, e-print arXiv: hep-ph/0801.1980.

4. L. Brink, P. Di Vecchia, P. Howe, Nucl.Phys. B118, (1977) 76.

5. Yu. S. Kalashnikova, A. Nefediev, Yu. A. Simonov, Phys. Rev. D 64, (2001) 014037.

6. Yu. A. Simonov, Phys. Atom. Nucl. 66, (2003) 338 [Yad. Fiz. 66, (2003) 363].

7. A. Yu. Dubin, A .B. Kaidalov, Yu .A .Simonov, Phys. Lett. B323, (1994) 41; Phys. Lett. B343, (1995) 310.

8. C. Semay, B. Silvestre-Brac, and I. Narodetskii, Phys. Rev. D 69, (2004) 014003, e-print ArXiv: hep-ph/0309256

9. F. Buisseret and V. Mathieu, Eur. Phys. J. A 29, (2006) 343.

10. P. Nunberg, D. Prosperi, and E. Pace, Nucl. Phys. A 285, (1977) 58 .

11. B. Silvestre-Brac, R. Bonnaz, C. Semay, and F. Brau, Quantum three-body problems using harmonic oscillator bases with different sizes, ISN Grenoble, ISN-00-66, 2000 (unpublished).

12. I. M. Narodetskii and M .A .Trusov, Phys. Atom. Nucl. 65, (2002) 917 [Yad. Fiz. 65, (2002) 949]; Phys. Atom. Nucl. 67, (2004) 762 [Yad. Fiz. 67, (2004) 783].

13. O. N. Driga, I. M. Narodetskii, A. I. Veselov, Phys. Atom. Nucl. 71, (2008) 335 [Yad. Fiz. 71, (2008) 356], e-print ArXiv: hep-ph/0712.1479.

14. Yu. A. Simonov, Phys. Lett. B 515, (2001) 137; A. DiGiacomo and Yu. A. Simonov, Phys. Lett. B 595, (2001) 368.

15. J. Carlson, J. Kogut, and W. R. Pandharipande, Phys. Rev. D 27, (1983) 233.

16. R. Sartor and F. Stancu, Phys. Rev. D 31, (1985) 128; Ibid, D 33, (1986) 727.

17. M. Fabre de la Ripelle and Yu. A. Simonov, Ann. Phys. (N.Y.) 212, (1991) 235.

18. N. Isgur, and G. Karl, Phys. Rev. D 18, (1978) 4187; K.-T. Chao, N. Isgur, and G. Karl, Phys. Rev. D 23, (1981)155.

19. B. Silvestre-Brac et al., Eur. Phys. J. C 32, (2004) 385.

20. Particle Data Group, W. -M. Yao et al., J. Phys. G 33, (2006)1. 
Table 1. Comparison of the eigenvalues $E_{0}$ of the Hamiltonian $H_{0}+V$ in Eq. (1) for the baryon ground states and the $\rho$ and $\lambda$ excitations obtained from the hyperspherical solution of Eq. (23) $\left(E_{0}^{Y}\right)$ and variational solution $\left(E_{0}^{\mathrm{M}}\right.$ var $)$. See the text for further explanation.

\begin{tabular}{|c|c|c|c|c|c|c|c|c|c|}
\hline Baryon & $L$ & Excitation & $\mu_{1}$ & $\mu_{3}$ & $E_{0}^{Y}$ & $E_{0}^{\mathrm{CM} M}$ & $E_{0}^{\Delta}$ & $E_{0}^{\mathrm{M}}$ & $E_{0}^{\mathrm{M}}$ \\
\hline$n n n$ & $\begin{array}{l}0 \\
1\end{array}$ & $\rho, \lambda$ & $\begin{array}{l}408 \\
457\end{array}$ & $\begin{array}{l}408 \\
457\end{array}$ & $\begin{array}{l}1318 \\
1638\end{array}$ & $\begin{array}{l}1366 \\
1697\end{array}$ & $\begin{array}{l}1230 \\
1532\end{array}$ & $\begin{array}{l}1299 \\
1615\end{array}$ & $\begin{array}{l}1297 \\
1612\end{array}$ \\
\hline nns & $\begin{array}{l}0 \\
1 \\
1\end{array}$ & $\begin{array}{l}\rho \\
\lambda\end{array}$ & $\begin{array}{l}414 \\
482 \\
441\end{array}$ & $\begin{array}{l}453 \\
459 \\
534\end{array}$ & $\begin{array}{l}1291 \\
1611 \\
1614\end{array}$ & $\begin{array}{l}1339 \\
1670 \\
1676\end{array}$ & $\begin{array}{l}1204 \\
1506 \\
1508\end{array}$ & $\begin{array}{l}1272 \\
1589 \\
1593\end{array}$ & $\begin{array}{l}1271 \\
1587 \\
1591\end{array}$ \\
\hline ssn & $\begin{array}{l}0 \\
1 \\
1\end{array}$ & $\begin{array}{l}\rho \\
\lambda\end{array}$ & $\begin{array}{l}458 \\
520 \\
483\end{array}$ & $\begin{array}{l}419 \\
424 \\
506\end{array}$ & $\begin{array}{l}1266 \\
1592 \\
1588\end{array}$ & $\begin{array}{l}1313 \\
1653 \\
1646\end{array}$ & $\begin{array}{l}1181 \\
1487 \\
1485\end{array}$ & $\begin{array}{l}1248 \\
1571 \\
1567\end{array}$ & $\begin{array}{l}1248 \\
1569 \\
1566\end{array}$ \\
\hline
\end{tabular}

Table 2. Comparison of baryon masses calculated using the AF approach and SSE. The symbol $\nu_{i}$ denotes either the constituent quark masses $\mu_{i}$ or the average kinetic energies of the current quarks $\omega_{i}$. Shown are the masses $M_{0}^{A F}$ and $M_{0}^{S S E}$ without the self-energy corrections, the self-energy corrections $C^{A F}$ and $C^{S S E}, M=M_{0}+C$ (all in units of MeV), and the relative error $\varepsilon$ defined by Eq. (33).

\begin{tabular}{|c|c|c|c|c|c|c|c|c|c|}
\hline Baryon & $\mathrm{L}$ & Excitation & Method & $\nu_{1}=\nu_{2}$ & $\nu_{3}$ & $M_{0}$ & $\mathrm{C}$ & M & $\varepsilon(\%)$ \\
\hline nnn & 0 & & $\begin{array}{c}\mathrm{AF} \\
\mathrm{SSE}\end{array}$ & $\begin{array}{l}408 \\
394\end{array}$ & $\begin{array}{l}408 \\
394\end{array}$ & $\begin{array}{l}1911 \\
1788\end{array}$ & $\begin{array}{l}-702 \\
-726\end{array}$ & $\begin{array}{l}1209 \\
1062\end{array}$ & 13.8 \\
\hline nns & 0 & & $\begin{array}{c}\mathrm{AF} \\
\mathrm{SSE}\end{array}$ & $\begin{array}{l}414 \\
396\end{array}$ & $\begin{array}{l}453 \\
484\end{array}$ & $\begin{array}{l}1946 \\
1877\end{array}$ & $\begin{array}{l}-648 \\
-657\end{array}$ & $\begin{array}{l}1298 \\
1220\end{array}$ & 6.4 \\
\hline ssn & 0 & & $\begin{array}{c}\mathrm{AF} \\
\mathrm{SSE}\end{array}$ & $\begin{array}{l}458 \\
404\end{array}$ & $\begin{array}{l}419 \\
465\end{array}$ & $\begin{array}{l}1182 \\
1904\end{array}$ & $\begin{array}{l}-598 \\
-602\end{array}$ & $\begin{array}{l}1384 \\
1302\end{array}$ & 6.3 \\
\hline nnn & 1 & $\begin{array}{l}\rho, \lambda \\
\rho, \lambda\end{array}$ & $\begin{array}{c}\mathrm{AF} \\
\mathrm{SSE}\end{array}$ & $\begin{array}{l}457 \\
440\end{array}$ & $\begin{array}{l}457 \\
440\end{array}$ & $\begin{array}{l}2301 \\
2186\end{array}$ & $\begin{array}{l}-627 \\
-651\end{array}$ & $\begin{array}{l}1674 \\
1534\end{array}$ & 9.1 \\
\hline nns & 1 & $\begin{array}{l}\rho \\
\rho \\
\lambda \\
\lambda\end{array}$ & $\begin{array}{c}\mathrm{AF} \\
\mathrm{SSE} \\
\mathrm{AF} \\
\mathrm{SSE}\end{array}$ & $\begin{array}{l}482 \\
464 \\
441 \\
415\end{array}$ & $\begin{array}{l}459 \\
465 \\
534 \\
592\end{array}$ & $\begin{array}{l}2356 \\
2245 \\
2330 \\
2315\end{array}$ & $\begin{array}{l}-581 \\
-594 \\
-592 \\
-603\end{array}$ & $\begin{array}{l}1751 \\
1652 \\
1738 \\
1712\end{array}$ & $\begin{array}{l}6.0 \\
1.5\end{array}$ \\
\hline ssn & 1 & $\begin{array}{l}\rho \\
\rho \\
\lambda \\
\lambda\end{array}$ & $\begin{array}{c}\mathrm{AF} \\
\mathrm{SSE} \\
\mathrm{AF} \\
\mathrm{SSE}\end{array}$ & $\begin{array}{l}520 \\
478 \\
483 \\
503\end{array}$ & $\begin{array}{l}424 \\
530 \\
506 \\
391\end{array}$ & $\begin{array}{l}2362 \\
2302 \\
2367 \\
2295\end{array}$ & $\begin{array}{l}-552 \\
-564 \\
-540 \\
-545\end{array}$ & $\begin{array}{l}1810 \\
1738 \\
1827 \\
1750\end{array}$ & $\begin{array}{l}4.1 \\
4.4\end{array}$ \\
\hline
\end{tabular}


